\title{
Artroo Avaliação do Sistema de Vigilância do Programa Nacional de Imunizações - Módulo Registro do Vacinado, Brasil, 2017
}

doi: 10.1590/\$1679-49742021000100028

\author{
Evaluation of the National Immunization Program Surveillance System - Vaccination Record \\ Module, Brazil, 2017
}

\section{Evaluación del Sistema de Vigilancia del Programa Nacional de Inmunizaciones - Módulo Registro del Vacunado, Brasil, 2017}

\author{
Aline Almeida da Silva ${ }^{1}$ - (1) orcid.org/0000-0001-9591-3774 \\ Antonia Maria da Silva Teixeira' - (1) orcid.org/0000-0002-9196-6331 \\ Carla Magda Allan Santos Domingues ${ }^{1}$ - (D) orcid.org/0000-0003-1463-4939 \\ Rui Moreira Braz' - (1) orcid.org/0000-0002-3834-578X \\ Cibelle Mendes Cabral' - • orcid.org/0000-0003-1934-3081
}

'Ministério da Saúde, Secretaria de Vigilância em Saúde, Brasília, DF, Brasil

\section{Resumo}

Objetivo: Avaliar o Sistema de Vigilância em Imunizações considerando-se o módulo Registro do Vacinado, do Sistema de Informações do Programa Nacional de Imunizações, Brasil, 2017. Métodos: Estudo descritivo, utilizando-se do Guidelines for Evaluating Public Health Surveillance Systems, publicado pelo Centers for Disease Control and Prevention (CDC/Atlanta/ GA/United States), para avaliar os atributos de simplicidade, flexibilidade, qualidade dos dados, sensibilidade, oportunidade e utilidade do sistema para seis vacinas do calendário de vacinação da criança. Resultados: 0 Sistema de Vigilância em Imunizações foi considerado complexo em sua descrição, flexível às mudanças no calendário vacinal, de baixa qualidade dos dados para as vacinas DTP e rotavírus, de aceitabilidade regular, com alta sensibilidade para a vacina BCG, inoportuno para a vacina contra hepatite B e útil às finalidades do Programa Nacional de Imunizações. Conclusão: Qualidade dos dados, aceitabilidade e oportunidade não apresentaram resultados satisfatórios, sendo necessárias ações pelo aprimoramento do sistema de informações.

Palavras-chave: Imunização; Programa Nacional de Imunizações; Vigilância em Saúde Pública; Avaliação de Programas e Projetos de Saúde; Sistemas de Informação; Qualidade dos Dados.

\section{Endereço para correspondência:}

Aline Almeida da Silva - Ministério da Saúde, Secretaria de Vigilância em Saúde, Programa de Treinamento em Epidemiologia de Campo Aplicada aos Serviços do SUS, QMSW 5, Lote 6, Condomínio Boulevard Antares I, Bloco 2, Apto. 322 , Sudoeste, Brasília, DF, Brasil. CEP: 70680-524

E-mail: aline.almeida@saude.gov.br 


\section{Introdução}

0 Programa Nacional de Imunizações (PNI) tem como missão a organização da política nacional de vacinação no país, ou seja, estruturação e coordenação das ações de imunização, monitoramento dos imunobiológicos e seus efeitos na população, visando reduzir a morbimortalidade decorrente de doenças imunopreveníveis. ${ }^{1-3}$

\section{Sistema de informações do Porgrama Nacional de Imunizações tem como finalidades (i) unificar sistemas paralelos, (ii) promover sua intercomunicação e (iii) disponibilizar dados individualizados da população vacinada.}

A gestão da informação do Sistema de Vigilância em Imunizações é feita por diversos sistemas de informações, abarcando outras áreas técnicas, dentro e fora do Ministério da Saúde. A respeito dos vacinados, utilizase o Sistema de Informações do Programa Nacional de Imunizações (SI-PNI), ${ }^{4}$ que possui três principais módulos: Registro do Vacinado, onde se encontram relatórios de cobertura vacinal e a listagem de faltosos, entre outros dados; Eventos Adversos Pós-Vacinação (EAPV); e Movimentação de Imunobiológicos (MI). Além destes módulos, o SI-PNI conta com informações agregadas, sobre as estratégias de multivacinação, monitoramento rápido de cobertura vacinal e campanhas. 0 SI-PNI tem como finalidades (i) unificar sistemas paralelos, (ii) promover sua intercomunicação e (iii) disponibilizar dados individualizados da população vacinada. ${ }^{5}$ Estes dados individualizados, sobre doses aplicadas, encontram-se no módulo Registro do Vacinado ${ }^{3}$ (Figura 1).

Para 0 monitoramento das ações do Sistema de Vigilância em Imunizações, é necessária a adesão ao SI-PNI e a alimentação regular e qualificada das informações pelos municípios. Os municípios que utilizam sistemas próprios, igualmente, devem informar dados no SI-PNI. A informação dos dados sobre imunização é feita pelos serviços que executam essa atividade, independentemente de sua natureza pública ou privada. ${ }^{6}$
Para incentivar a adesão dos municípios ao SI-PNI, destacam-se duas portarias publicadas pelo Ministério da Saúde: a Portaria GM/MS n ${ }^{0} 2.363$, de 18 de outubro de 2012, que instituiu o repasse financeiro do Fundo Nacional de Saúde (FNS) aos estados e municípios para aquisição de equipamentos de informática; e a Portaria GM/MS n ${ }^{0} 2.984$, de 27 de dezembro de 2016, que trata das metas e indicadores do Programa de Qualificação das Ações de Vigilância em Saúde (PQA-VS). A Portaria GM/MS n ${ }^{\circ}$ 2.984/2016 atribui ao PNI dois indicadores, um deles definindo pela seguinte meta: $\geq 80 \%$ das salas de vacina dos municípios com alimentação mensal do SI-PNI, justamente objeto do presente estudo. ${ }^{7,8}$

A necessidade de identificar as fragilidades e potencialidades do Sistema de Vigilância em Imunizações, de forma a qualificar as informações sobre imunizações no Brasil, escassez de publicações relacionadas ao SI-PNI e ausência de análise específica, faz necessário conhecer o módulo Registro do Vacinado, seu desempenho, consistências e possibilidades de melhoria.

Este estudo teve por objetivo avaliar o Sistema de Vigilância em Imunizações a partir da análise do módulo Registro do Vacinado, do SI-PNI, referente ao ano de 2017.

\section{Métodos}

Foi realizado um estudo descritivo e de avaliação do Sistema de Vigilância em Imunizações, com uso de dados secundários dos relatórios do módulo Registro do Vacinado, do SI-PNI, avaliado em todas as esferas do Sistema Único de Saúde (SUS), considerando-se as 27 Unidades da Federação (UFs), os 5.570 municípios e as 35.458 salas de vacina ativas no país, no ano de 2017, adotando-se, como referencial teórico, a publicação Guidelines for Evaluating Public Health Surveillance Systems, do Centers for Disease Control and Prevention dos Estados Unidos (CDC, Atlanta, GA, USA).?

Foram critérios de avaliação do Sistema de Vigilância em Imunizações seus atributos qualitativos - simplicidade, flexibilidade, qualidade dos dados, aceitabilidade - e quantitativos - sensibilidade, oportunidade e completude -, explicitados a seguir: 
a) Simplicidade

Avaliação subjetiva, por meio da descrição do Sistema de Vigilância em Imunizações, sendo a classificação final deste atributo como simples ou complexo.

b) Flexibilidade Adequação do Sistema de Vigilância em Imunizações (i) às mudanças de faixa etária e grupo-alvo da vacina contra o papilomavírus humano (HPV), (ii) ao esquema vacinal da febre amarela e (iii) à intercomunicabilidade entre os módulos Registro do Vacinado e Evento Adverso Pós-Vacinação. Utilizaram-se, como critérios, as mudanças no sistema de acordo com as alterações no calendário ('Sim' ou 'Não') e a Regra de Negócios - esta, considerada adequada ou inadequada, segundo a interoperabilidade ou não entre os módulos.

A classificação final deste atributo foi a de flexível (ao atingir três ou mais critérios positivos) ou pouco flexível (ao atingir menos de três critérios positivos).

c) Qualidade dos dados

Para avaliar a completitude do Sistema de Vigilância em Imunizações, utilizou-se do relatório 'Vacinados por Vacina' da hepatite B e suas variáveis 'nome da mãe', 'endereço', 'telefone' e 'lote da vacina'. A escala de classificação deste atributo foi definida da seguinte forma: para $\leq 5 \%$ de variáveis em branco, completitude boa; e para $>5 \%$, completitude ruim. Para inconsistência dos dados, utilizaram-se três relatórios do sistema: 'Erros de Registro', entre os quais se buscou encontrar apenas doses aplicadas da vacina contra difteria, tétano e coqueluche (pertussis) (DTP) fora da faixa etária preconizada; 'Listagem de Faltosos' da vacinação contra o rotavírus, em que se investigaram, na variável 'dias de atraso', apenas atrasos $>30$ dias; e 'Vacinados por Vacina', para a $1^{\text {a }}$ e a $2^{a}$ doses da vacina do rotavírus, em que se buscou encontrar, nas doses fora da faixa etária-limite preconizada, o campo 'Dose Inadvertida' igual a 'Sim'. A Regra de Negócios foi classificada como adequada ou inadequada.

A classificação final da qualidade de dados foi a seguinte: alta, moderada ou baixa, a partir da série de combinações entre a completitude e a inconsistência (Figura 2). d) Aceitabilidade

Avaliou-se a adesão das salas de vacina dos municípios ao SI-PNI, considerando-se as portarias ministeriais citadas.

A classificação final sobre este atributo foi a seguinte: excelente, para $\geq 80 \%$ das salas de vacina utilizando o sistema; regular, entre $\geq 50$ e $<80 \%$; ou ruim, quando $<50 \%$ das salas utilizavam 0 SI-PNI.

e) Sensibilidade

Considerou-se as crianças menores de 1 ano captadas para vacinação com BCG (bacilo de Calmette-Guérin) em 2017, em relação aos nascidos vivos registrados no Sistema de Informações sobre Nascidos Vivos (Sinasc) em 2016.

0 critério utilizado para a classificação deste atributo foi o seguinte: sensibilidade alta, quando $\geq 80 \%$ das crianças nessa idade foram vacinadas com BCG; ou sensibilidade baixa, quando $<80 \%$ dessas crianças foram imunizadas pela vacina.

f) Oportunidade

Oportunidade de vacinação contra hepatite B no prazo de até um dia de vida, em relação às doses aplicadas até 30 dias de vida.

0 critério utilizado nesta classificação foi 0 seguinte: sistema oportuno, para $\geq 80 \%$ das doses feitas em até um dia de vida; ou sistema inoportuno, para $<80 \%$ das doses aplicadas nesse prazo de tempo.

g) Utilidade

Visando estimar o cumprimento da finalidade do Sistema de Vigilância em Imunizações, a avalição deste atributo pautou-se na construção de dados de importância para a orientação e tomada de decisão da Saúde Pública. Entre outros aspectos, a utilidade considera a usabilidade e os efeitos dos sistemas nas decisões sobre políticas públicas. ${ }^{9}$ Embora seja um componente da avaliação de sistemas, a utilidade pode sofrer influências dos atributos, segundo esta metodologia de avaliação.

Para avaliação da completitude e oportunidade do Sistema de Vigilância em Imunizações, dados os erros observados na exportação dos relatórios das capitais, utilizou-se como critério de substituição o município mais populoso de cada UF, segundo o Instituto Brasileiro de Geografia e Estatística (IBGE), e assim sucessivamente, até que um relatório fosse exportado. 

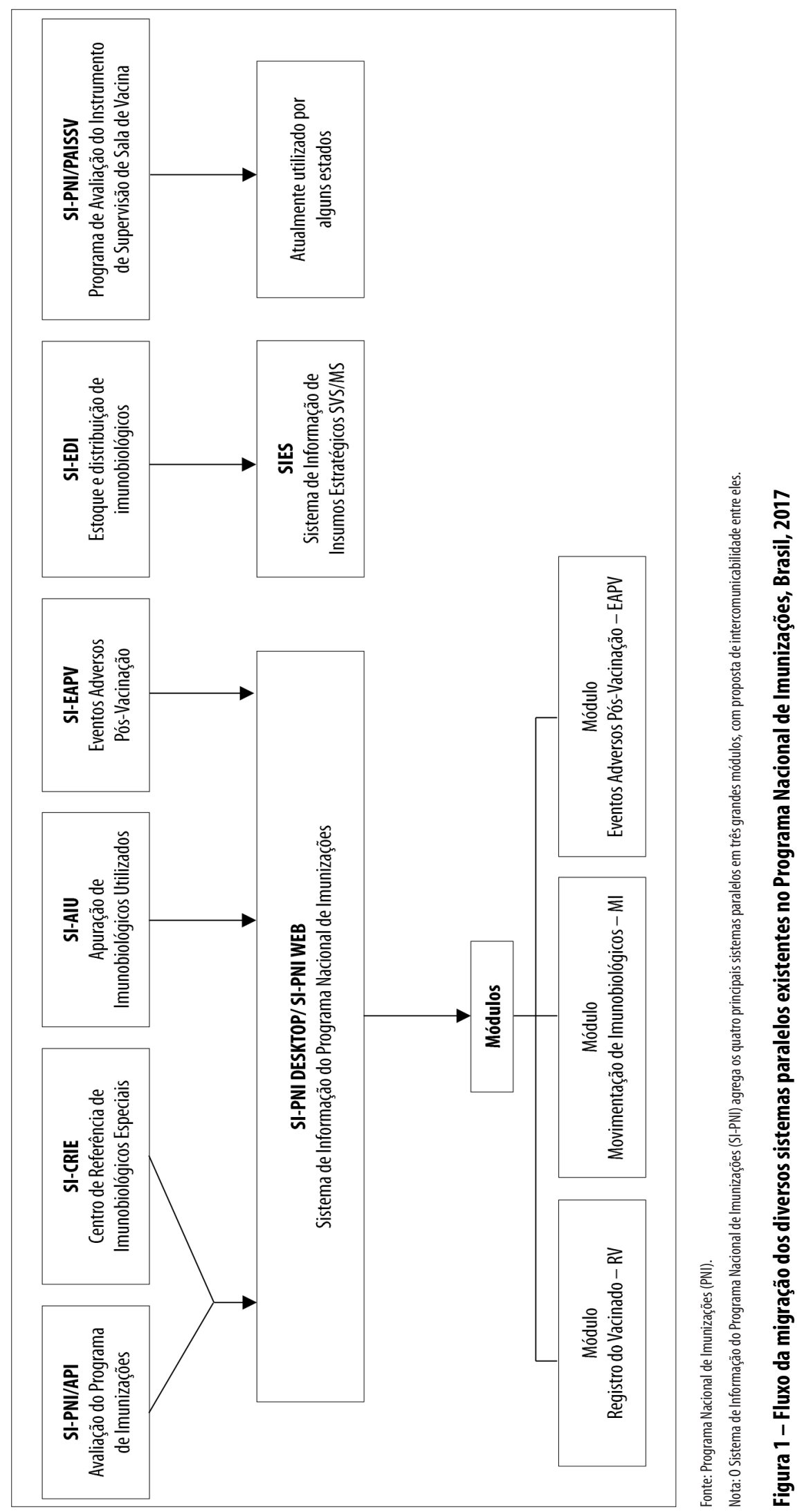


\begin{tabular}{|c|c|c|}
\hline Atributo & \multicolumn{2}{|l|}{ Classificação/pontos de corte } \\
\hline Simplicidade & Simples ou complexo & $\begin{array}{l}\text { Avaliado de forma subjetiva, considerando a descrição do Sistema de } \\
\text { Vigilância em Imunizações. }\end{array}$ \\
\hline Flexibilidade & Flexível ou pouco flexível & $\begin{array}{l}\text { Avaliado se o sistema de informação se adequou às mudanças do } \\
\text { Calendário Vacinal. Como ponto de corte, consideram-se três ou mais } \\
\text { critérios positivos. }\end{array}$ \\
\hline \multirow{6}{*}{ Qualidade dos dados } & Alta & $\begin{array}{l}\text { Completitude Boa; Regra de Negócios classificada como adequada nos } \\
\text { três quesitos de inconsistência. }\end{array}$ \\
\hline & \multirow{2}{*}{ Moderada } & $\begin{array}{l}\text { Completitude Boa; Regra de Negócios classificada como adequada em } \\
\text { um ou dois quesitos de inconsistência. }\end{array}$ \\
\hline & & $\begin{array}{l}\text { Completitude Ruim; Regra de Negócios classificada como adequada em } \\
\text { dois ou três quesitos de inconsistência. }\end{array}$ \\
\hline & \multirow{3}{*}{ Baixa } & $\begin{array}{l}\text { Completitude Boa; Regra de Negócios classificada como inadequada nos } \\
\text { três quesitos de inconsistência. }\end{array}$ \\
\hline & & $\begin{array}{l}\text { Completitude Ruim; Regra de Negócios classificada como adequada em } \\
\text { apenas um quesito de inconsistência. }\end{array}$ \\
\hline & & $\begin{array}{l}\text { Completitude Ruim; Regra de Negócios classificada como inadequada } \\
\text { nos três quesitos de inconsistência. }\end{array}$ \\
\hline Aceitabilidade & Excelente, regular ou ruim & $\begin{array}{l}\text { Avaliou-se a adesão das salas de vacina ao SIPNI. Os cortes foram: } \\
\geq 80 \% ; \geq 50 \text { a }<80 \% ; \mathrm{e}<50 \% \text {. }\end{array}$ \\
\hline Sensibilidade & Alta ou baixa & $\begin{array}{l}\text { Crianças menores de } 1 \text { ano de idade, captadas para vacinação com BCG, } \\
\text { em relação aos nascidos vivos. } 0 \text { corte foi de } 80 \% \text {. }\end{array}$ \\
\hline Oportunidade & Oportuno ou inoportuno & $\begin{array}{l}\text { Oportunidade de vacinação com a hepatite B em até } 1 \text { dia de vida em } \\
\text { relação às doses aplicadas até } 30 \text { dias de vida. } 0 \text { corte foi de } 80 \% \text {. }\end{array}$ \\
\hline Utilidade & $\begin{array}{l}\text { Não foi feita uma classificação deste componente } \\
\text { da avaliação, pois considera-se que os sistemas } \\
\text { são uteis, porém, passíveis de melhoria. }\end{array}$ & Foi avaliado por meio da finalidade do sistema. \\
\hline
\end{tabular}

\section{Figura 2 - Classificação final do atributo de qualidade dos dados, segundo a combinação de resultados de completitude} e inconsistência

Para os estados da Bahia, Ceará, Goiás, Mato Grosso do Sul, Minas Gerais, Pará, Paraná, Santa Catarina e São Paulo, foram utilizados como referência os seguintes municípios, respectivamente: Feira de Santana (BA), Caucaia (CE), Aparecida de Goiânia (G0), Dourados (MS), Contagem (MG), Ananindeua (PA), Londrina (PR), Joinville (SC) e Santo André (SP).

Foram analisadas as seguintes vacinas, com seus critérios de escolha: ${ }^{: 10-13}$

a) Vacinas BCG e hepatite B: indicadas ao nascer A BCG é considerada a 'porta de entrada' da criança no Sistema de Vigilância em Imunizações, sendo recomendada até $<5$ anos de idade.

A vacina da hepatite B é indicada até os 30 dias de vida; preferencialmente, no primeiro dia de vida, ainda na maternidade, visando reduzir o risco de transmissão vertical da infecção. b) Vacinas do rotavírus humano e DTP: restrição de faixa etária-limite para vacinação

A vacina do rotavírus humano é indicada aos 2 e aos 4 meses de vida, tendo como prazos-limite: $1^{\mathrm{a}}$ Dose - a ser aplicada no período de $\geq 1$ mês e 15 dias a $\leq 3$ meses e 15 dias; e

$2^{\text {a }}$ Dose - a ser aplicada no período de $\geq 3$ meses e 15 dias a $\leq 7$ meses e 29 dias.

Trata-se de prazos a serem cumpridos impreterivelmente, dado o risco de invaginação intestinal. Já a vacina DTP é indicada para aplicação no prazo de $\geq 2$ meses a $<7$ anos, devido ao componente pertussis, que pode ocasionar agravamento de eventos adversos. Esta vacina, utilizada como reforço da vacina conhecida como pentavalente (DTP + Haemophilus influenzae B + hepatite B), é recomendada aos 15 meses de vida e aos 4 anos de idade. 


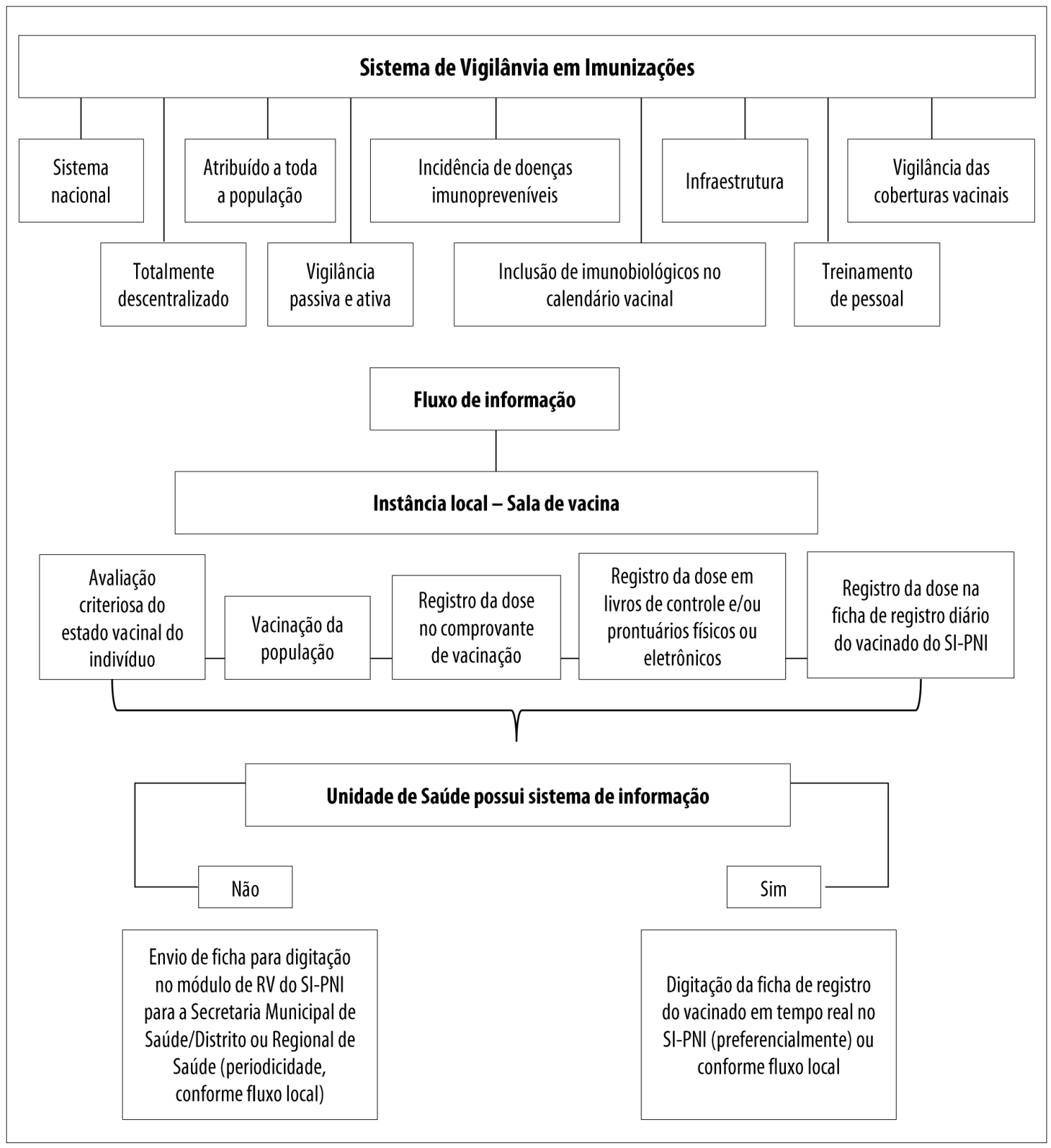

Figura 3 - 0 Sistema de Vigilância em Imunizações e o fluxo de informações dos dados de vacinação, desde a aplicação da vacina até a alimentação da informação no sistema 
c) Vacinas da febre amarela e HPV: mudanças no calendário vacinal em 2017

Para a vacina da febre amarela, até 2016, cumpria-se o esquema de uma dose e um reforço a cada dez anos. A partir de 2017, passou-se a prescrever:

Dose Única - para toda a vida; e

Dose Fracionada - estratégia temporária, em alguns municípios prioritários.

A rotina da vacinação contra 0 HPV, por sua vez, não apenas ampliou a faixa etária das meninas-alvo, de 9 a 13 anos para 9 a $<15$ anos, mas também incluiu meninos de $11 \mathrm{a}<15$ anos.

As fontes de dados do estudo consistiram dos relatórios do SI-PNI, manuais técnicos e operacionais do PNI, dados do Sinasc e do IBGE. Para análise dos dados, utilizou-se o Microsoft Excel 2013.

\section{Resultados}

\section{Simplicidade}

0 Sistema de Vigilância em Imunizações é um sistema nacional, totalmente descentralizado, responsável pela efetividade na imunização de toda a população, consideradas as especificidades de grupos-alvo, indicações clínicas e estratégias de prevenção e controle de doenças, consoante o contexto epidemiológico (Figura 3). A vacinação configura-se como ação de vigilância passiva (demanda espontânea das salas de vacina) e ativa (busca de não vacinados), e para tanto, envolve as três esferas de gestão, organizadas que em instâncias: nacional; estadual; regional; municipal; e local.

0 Sistema de Vigilância em Imunizações avalia a incidência de doenças imunopreveníveis, sua morbimortalidade e deteç̧ão de suscetibilidade, estando diretamente integrada à Vigilância Epidemiológica mais ampla. ${ }^{14}$ Para a decisão sobre mudanças no calendário vacinal, é realizado monitoramento dos resultados de pesquisas nacionais e internacionais, tendo como foro de discussão o Comitê Técnico Assessor em Imunizações, organismo de caráter consultivo, composto por técnicos do PNI, pesquisadores e especialistas, entre outros. ${ }^{1,15}$

Laboratórios nacionais e internacionais podem ser incluídos no processo, buscando-se o fortalecimento do complexo industrial, mediante transferências de tecnologia, e a habilitação do Brasil na produção sustentável desses imunobiológicos. ${ }^{15}$

Uma vez incluída a mudança na rotina, o Sistema de Vigilância em Imunizações exige infraestrutura adequada para o recebimento dos imunobiológicos, do nível federal à sala de vacina local (armazenamento, controles de estoque, registro das informações), além de pessoal capacitado para 0 apoio técnico e a execução de todos os processos, incluindo 0 ato da vacinação, $\mathrm{e}$ habilidade no uso das ferramentas de registro. ${ }^{6}$

0 Sistema de Vigilância em Imunizações conta com a Ficha do Registro Diário do Vacinado e seus 25 macrocampos. A depender das indicações e quantidades de vacinas a serem administradas, aumenta a quantidade de registros. Caso a unidade de saúde disponha do sistema, a digitação deve ocorrer na sala de vacina, preferencialmente com o vacinado e/ou seu responsável presente; caso contrário, a ficha preenchida deve ser encaminhada para digitação em outra localidade, conforme o fluxo local estabelecido. Unidades dotadas de sistemas próprios devem transferir os dados para o SI-PNI. ${ }^{16}$

0 SI-PNI possui 14 menus principais e 147 submenus; destes, 81 são relatórios, sendo 59 do módulo Registro do Vacinado. Todas as instâncias têm acesso ao sistema, embora apenas os estabelecimentos de saúde realizem entrada de dados. Destaca-se a intercomunicação do SI-PNI com o Cartão Nacional do SUS, para importação de dados de identificação do usuário. ${ }^{4}$

As Regras de Negócios, incluídas nesta análise, são um conjunto de critérios definidos pelo PNI, baseados em informações técnico-científicas e indicações para cada imunobiológico, e, assim, servem de referência para as críticas ao sistema na entrada e saída do dado. Trata-se de uma planilha com 24 colunas e 83 linhas, para descrição de vacinas, diluentes, soros e imunoglobulinas, indicações de aplicação e custo. Cada imunobiológico conta com uma Regra de Negócios específica, de acordo com a história natural da doença cuja infecção ele previne.

0 monitoramento das Regras de Negócios é realizado pela área técnica de informação do PNI, rotineira e sistematicamente. Na identificação de erros, os ajustes recomendados são solicitados ao Departamento de Informática do SUS (Datasus). 
Considerando-se (i) os vários eixos de atuação, (ii) os diversos profissionais e entidades envolvidos nesses processos, (iii) a condição de profissional habilitado e treinado para as ações de imunização ${ }^{6}$ e (iv) a exigência de monitoramento contínuo e sistemático, o Sistema de Vigilância em Imunizações foi classificado complexo.

\section{Flexibilidade}

0 Sistema de Vigilância em Imunizações, relativamente à vacina contra $0 \mathrm{HPV}$, mostrou-se adequado à incorporação de uma nova estratégia, por faixa etária (registro: 'Sim'), e à inclusão de um novo público-alvo (registro: 'Sim'). 0 sistema também se mostrou adequado à prescrição de um novo esquema vacinal da febre amarela (registro: 'Sim'); já a interoperabilidade dos módulos Registro do Vacinado e Eventos Adversos Pós-Vacinação revelou-se inadequada (registro: 'Não').

Por conseguinte, obteve-se um escore final de três critérios positivos para este atributo, sendo o sistema classificado flexível.

\section{Qualidade dos dados}

Ao se avaliar a completitude no registro dos dados 'nome da mãe', 'endereço' e 'telefone', observou-se que o relatório não exporta essas variáveis, impossibilitando a avaliação deste atributo do sistema. Para análise da variável 'lote da vacina', foram avaliados 696.593 registros, dos quais 38.219 (5,5\%) se encontravam em branco, pelo que a completitude dos dados foi considerada ruim.

Quanto à inconsistência, no relatório 'Erros de Registro', dos 112.333 registros, foram encontrados 27.787 dentro da faixa etária permitida, sendo a Regra de Negócios do SI-PNI classificada como inadequada sob o aspecto dos erros identificados nos registros.

No relatório 'Listagem de Faltosos', dos 3.852 registros, 228 estavam com 30 dias ou mais de atraso, e aqui também, a Regra de Negócios foi classificada como inadequada.

No relatório 'Vacinados por Vacina', do total de 4.868 registros, 2.656 eram de primeira dose, contendo 325 registros fora da faixa etária de eleição. Destes, 185 apresentavam o registro 'Não' no campo 'Dose Inadvertida'. Da segunda dose (2.212 registros), foram encontrados 136 registros fora da faixa etária de eleição, e destes, 82 apresentavam 'Não' no campo
'Dose Inadvertida'. A Regra de Negócios também foi classificada como inadequada quanto à observação dos vacinados por vacina.

Portanto, a classificação final do atributo foi de baixa qualidade dos dados.

\section{Aceitabilidade}

Dos 5.570 municípios brasileiros, 569 possuíam menos de $50 \%$ das suas salas de vacina utilizando o SI-PNI; e 762 municípios contavam com 50 a $<80 \%$ de suas salas de vacina ativas. A baixa adesão ao Sistema de Vigilância em Imunizações em 2017 foi observada, principalmente, nos estados de Roraima e Rio de Janeiro, e em alguns municípios da região Nordeste.

0 sistema era utilizado $\mathrm{em} \geq 80 \%$ das salas de vacina de 4.239 (76\%) municípios, sendo sua aceitabilidade classificada como regular (Figura 4).

\section{Sensibilidade}

Foram registradas no SI-PNI 2.708.944 doses da vacina contra o BCG em crianças menores de 1 ano, em 2017; de acordo com o Sinasc, houve 2.857.800 nascidos vivos em 2016.

Como 94,8\% das crianças que constam na base de dados do Sinasc foram captadas, o sistema foi classificado como de alta sensibilidade.

\section{oportunidade}

Foram administradas 134.531 doses da vacina contra hepatite $\mathrm{B}$ em $\leq 30$ dias de vida; dessas doses, 106.609 foram aplicadas em $\leq 1$ dia de vida, resultando em um percentual de $79,2 \%$.

Assim, o Sistema de Vigilância em Imunizações foi considerado inoportuno (Tabela 1).

\section{Utilidade}

0 Sistema de Vigilância em Imunizações revelouse útil no cumprimento de sua finalidade, permitindo identificar a população suscetível às doenças imunopreveníveis por dados individualizados e a gestão de ações oportunas no território. 0 sistema não só ofereceu informações sobre 0 calendário vacinal disponível na rede pública, mas também ampliou a oferta de vacinas ao longo dos anos, contemplando diversos grupos etários, e mostrou-se capaz de agrupar seus subsistemas, embora ainda necessite de ajustes nas funcionalidades. 


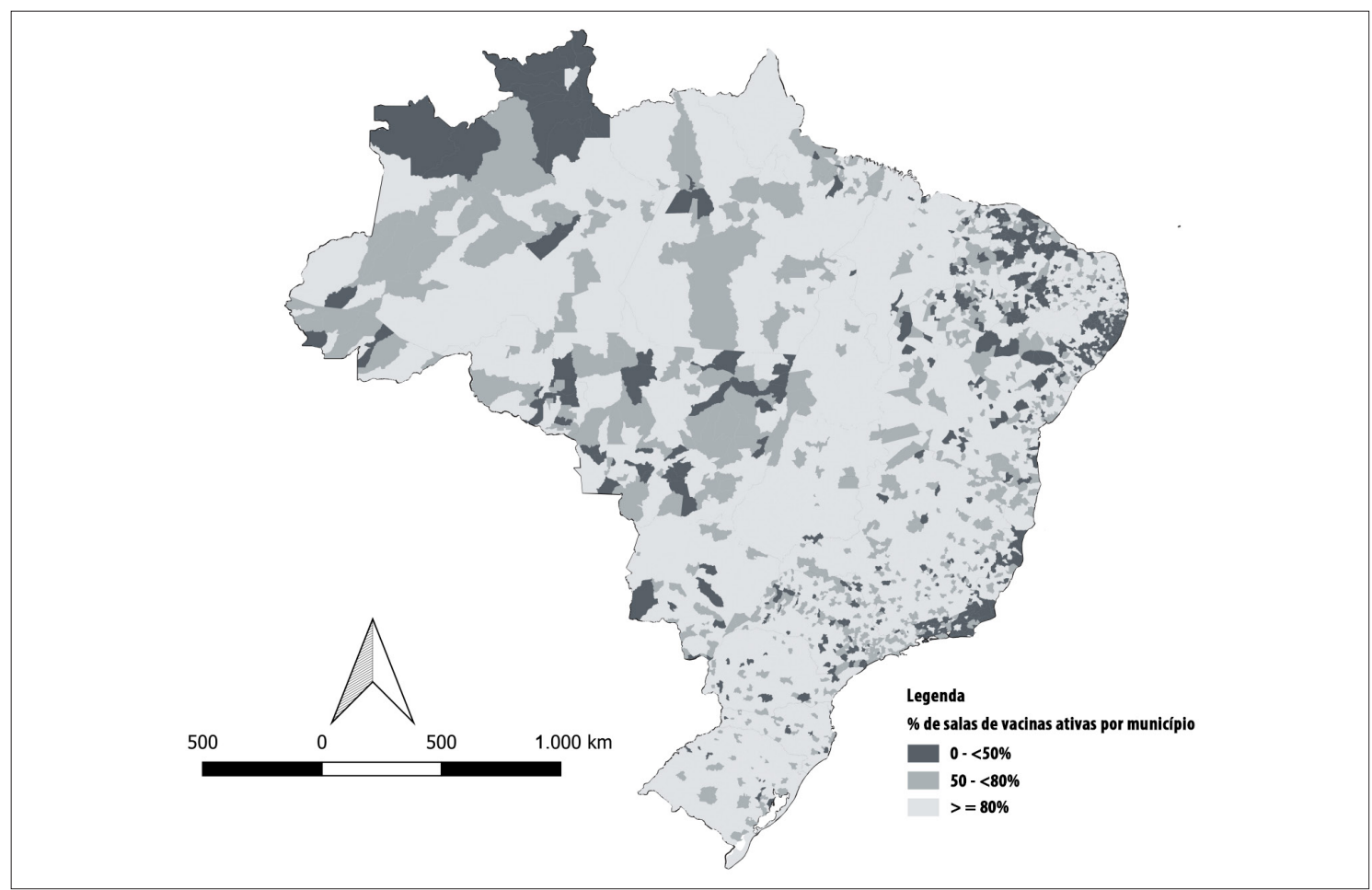

Figura 4 - Aceitabilidade dos municípios quanto à adesão ao Sistema de Informação do Programa Nacional de Imunizações, considerando-se $\geq 80 \%$ das salas de vacina a utilizá-lo, Brasil, 2017

\section{Discussão}

0 Sistema de Vigilância em Imunizações contribui para o controle, eliminação ou erradicação de doenças imunopreveníveis no território nacional. Sua avaliação, considerando-se as seis vacinas do Calendário Nacional de Vacinação do PNI, apontou que o sistema é complexo, flexível, de baixa qualidade quanto aos dados para as vacinas DTP e rotavírus, de baixa aceitabilidade, alta sensibilidade para a BCG, inoportuno para a vacina contra a hepatite $B$, e útil, no cumprimento de sua finalidade.

Este estudo observou que a complexidade do Sistema de Vigilância em Imunizações se deve, em parte, à necessidade de monitoramento do perfil epidemiológico das doenças imunopreveníveis, à parceria com especialistas e pesquisadores e à estruturação dos serviços de saúde com logística e infraestrutura adequadas, e profissionais habilitados e treinados para executar as ações de vacinação. ${ }^{3,16} \mathrm{~A}$ vigilância em imunizações exige 0 envolvimento de diversos setores e níveis de atenção, em todos os seus processos. Ela depende, resulta de e fornece elevada quantidade de informações, o que lhe confere ao perfil tal complexidade. ${ }^{17}$

0 sistema foi considerado flexível, adequou-se às mudanças do calendário nacional de vacinação, anteriormente à implementação da mudança na rotina, propiciando ao operador o tempo hábil para apropriação e alimentação correta das variáveis do sistema. Segundo o Guidelines for Evaluating Public Health Surveillance Systems do CDC/US, a complexidade de um sistema tende a torná-lo inflexível, o que não foi observado nesta avaliação. ${ }^{9}$

A baixa qualidade dos dados, evidenciada pela inadequada completitude e consistência, impacta diretamente na validação, precisão e confiabilidade dos dados e outros aspectos inerentes à necessária qualificação das informações, ${ }^{18,19}$ podendo interferir na avaliação da real situação de saúde e, também, na viabilidade de comparação com outras bases de dados. ${ }^{20}$ No que concerne à baixa completitude da variável 'lote da vacina', este achado pode estar relacionado ao fato 
de não se tratar de variável obrigatória, conquanto seu preenchimento pudesse auxiliar na identificação de surtos e/ou eventos adversos pós-vacinação. ${ }^{21}$

Destaca-se, primeiramente, a importância da qualificação dos profissionais envolvidos na alimentação dos dados no sistema de informações, contribuindo para o fortalecimento da capacidade operacional dos municípios. Cabe, também, um esforço de capacitação permanente dos especialistas na área de Tecnologia da Informação, à altura de seu trabalho com as Regras de Negócio, na análise dos dados inseridos e exportados no sistema de informações. ${ }^{5,20}$

Quanto à baixa aceitabilidade, os municípios que não aderem ao sistema de informações podem ter dificuldade na gestão de sua informação, principalmente na avaliação do impacto das ações de vigilância, e no alcance de indicadores. ${ }^{22}$ Em contrapartida, é importante destacar as diversas realidades existentes para a acessibilidade de rede, especialmente em locais do país de difícil acesso, o que pode influenciar no desempenho desse atributo. ${ }^{23}$

0 sistema se mostrou sensível, haja vista a vigilância em imunizações ter captado um percentual satisfatório de crianças menores de 1 ano, acima do valor de referência, para a vacinação com BCG ao nascer. Atribui-se essa sensibilidade, ainda que parcialmente, à recomendação da vacinação ainda na maternidade, e, caso a criança não seja vacinada por ocasião do nascimento, à possibilidade de sua captação posterior, para vacinação. ${ }^{10}$

Embora 0 cálculo utilizado para a sensibilidade seja o mesmo da cobertura vacinal, o percentual de sensibilidade neste estudo difere do percentual de cobertura. 0 Guidelines for Evaluating Public Health Surveillance Systems do CDC/US permite considerar a sensibilidade em dois níveis: no primeiro, a proporção de eventos captados pela vigilância; e no segundo, a habilidade para descobrir surtos ou monitorar mudanças ao longo do tempo. ${ }^{9}$ Neste escopo, o cálculo proposto no estudo mostra-se adequado, pois identifica o percentual de crianças captadas para vacinação em relação às nascidas vivas.

Ressalta-se a divergência no resultado da captação para a vacinação com BCG e na oportunidade da vacinação contra hepatite $B$, uma vez que as duas vacinas são recomendadas simultaneamente. ${ }^{10}$ A inoportunidade do sistema na imunização contra hepatite $B$ chama a atenção para a necessidade de avaliação conjunta da oportunidade da vacina BCG, buscando identificar a suscetibilidade das crianças a essas doenças imunopreveníveis nos primeiros dias de vida. Deve-se considerar os períodos máximos de vacinação de ambas as vacinas.

A inoportunidade do Sistema de Vigilância em Imunizações para necessita ser discutida conjuntamente, com as esferas de gestão estadual e municipal, para solução dessa questão levantada pelo estudo, no sentido da descentralização do procedimento para 0 maior número de maternidades, ou, se necessário, da capacitação do profissional da assistência hospitalar para a resolução desse problema.

0 Sistema de Vigilância em Imunizações mostrouse útil, por cumprir com suas finalidades, permitir identificar fragilidades e potencialidades, e assim contribuir para a melhoria de sua qualidade, sobretudo com informação de bases técnicas sólidas, para subsidiar a tomada de decisão.

Este componente da avaliação está diretamente associado à relevância de sua finalidade, na relação direta entre o que se espera e o que é percebido das informações produzidas pelo sistema. ${ }^{18,21,24}$

Entre as principais limitações do estudo, encontram-se (i) 0 uso de dados secundários e de população do Sinasc 2016, para estimar indicadores de vacinação realizada no ano de 2017, podendo subestimar ou superestimar os dados, (ii) a consulta a grandes bancos de dados, impossibilitando a exportação de relatórios, e, assim, permitindo a análise de um menor quantitativo de registros, e (iii) a não utilização do SI-PNI por alguns municípios e, como consequência disso, o menor volume de registros para análise.

Embora o Sistema de Vigilância em Imunizações se revele complexo em sua estrutura, flexível, com baixa qualidade dos dados para as vacinas DTP e rotavírus, de baixa aceitabilidade, de alta sensibilidade para a vacina $\mathrm{BCG}$ e inoportuno para a vacina da hepatite $B$, é mister que sua avaliação seja feita periodicamente, para identificar possíveis falhas nos processos, prevenindo seu possível impacto no desempenho do PNI.

0 PNI expandiu-se, ao longo dos anos, na oferta de diversos imunobiológicos. ${ }^{13}$ Este avanço, tão expressivo, impõe o desenho de novas formas de gestão para o PNI, com base em informações mais consistentes e oportunas. A elaboração de estratégias conjuntas, com a participação dos entes federados, pode contribuir para a identificação e resolução dos nós críticos em 
Tabela 1 - Oportunidade do Sistema de Vigilância do Programa Nacional de Imunizações, segundo a idade para vacinação contra hepatite B, por capitais ou municípios do país selecionados, Brasil, 2017

\begin{tabular}{|c|c|c|c|}
\hline Município & Doses aplicadas $\leq 30$ dias & Dose oportuna $\leq 24 \mathrm{~h}$ & Oportunidade (\%) \\
\hline Rio Branco & 6.729 & 5.422 & 80,6 \\
\hline Maceió & 2.584 & 108 & 4,2 \\
\hline Macapá & 8.254 & 4.226 & 51,2 \\
\hline Parintins & 1.887 & 1.582 & 83,8 \\
\hline Feira de Santana & 4.827 & 2.083 & 43,2 \\
\hline Caucaia & 574 & 381 & 66,4 \\
\hline Brasília & 11.773 & 10.447 & 88,7 \\
\hline Vitória & 3.149 & 2.402 & 76,3 \\
\hline Aparecida de Goiânia & 1.094 & 375 & 34,3 \\
\hline São Luís & 204 & 14 & 6,9 \\
\hline Cuiabá & 442 & 256 & 57,9 \\
\hline Dourados & 3.092 & 2.540 & 82,1 \\
\hline Contagem & 4.924 & 2.588 & 52,6 \\
\hline Ananindeua & 2.787 & 1.735 & 62,3 \\
\hline João Pessoa & 1.804 & 915 & 50,7 \\
\hline Londrina & 3.738 & 3.706 & 99,1 \\
\hline Recife & 3.267 & 1.891 & 57,9 \\
\hline Teresina & 10.426 & 8.691 & 83,4 \\
\hline Rio de Janeiro & 21.103 & 19.201 & 91,0 \\
\hline Natal & 2.318 & 1.234 & 53,2 \\
\hline Porto Alegre & 4.956 & 4.850 & 97,9 \\
\hline Porto Velho & 4.718 & 3.757 & 79,6 \\
\hline Boa Vista & 5.192 & 5.127 & 98,7 \\
\hline Joinville & 12.340 & 11.999 & 97,2 \\
\hline Santo André & 6.624 & 6.374 & 96,2 \\
\hline Aracaju & 1.719 & 1.257 & 73,1 \\
\hline Palmas & 4.006 & 3.448 & 86,1 \\
\hline 27 municípios & 134.531 & 106.609 & 79,2 \\
\hline
\end{tabular}

Fonte: SI-PNI, Módulo Registro de Vacinado/GGPN/DEVIT/SVS/MS, acessado em 29/08/2018.

imunização, visando qualificar o planejamento, a organização e coordenação do Sistema de Vigilância em Imunizações. ${ }^{5,19}$

Nesse sentido, recomenda-se à Coordenação Nacional do PNI reorganizar os processos e fluxos de seu sistema de informações, visando ampliar a adesão ao Sistema de Vigilância em Imunizações, melhorar a qualidade de dados, aumentar a captação oportuna de indivíduos para vacinação e aperfeiçoar a integração do SI-PNI com as bases de dados de outros sistemas. ${ }^{5,16}$ É fundamental estimular gestores de estados e municípios a aderirem ao Sistema de Vigilância em Imunizações; e fortalecer as ações de treinamento e supervisão da rede de vigilância em imunizações in loco, com foco na melhoria da qualidade dos dados.

Para o SI-PNI cabe fomentar a aquisição de novos equipamentos de informática, capazes de processar grandes bancos de dados, e trabalhar com 
a equipe do Departamento de Informática do SUS (Datasus) na elaboração de soluções tecnológicas, inclusive a reformulação do sistema, com o objetivo de melhorar seu desempenho como um todo e torná-lo menos complexo para os operadores, em todas as suas instâncias.

Outrossim, cumpre averiguar a distribuição e oferta de vacina contra hepatite $B$, junto aos coordenadores estaduais e municipais, e de treinamento aos profissionais das unidades de saúde, para garantir a vacinação oportuna dos recém-nascidos. Recomenda-se, também, a avaliação periódica do programa, utilizando-se de diferentes metodologias.

\section{Referências}

1. Domingues CMAS, Teixeira AMS. Coberturas vacinais e doenças imunopreveníveis no Brasil no período 1982-2012: avanços e desafios do Programa Nacional de Imunizações. Epidemiol Serviços Saúde [Internet]. 2013 jan-mar [citado 2018 set 6];22(1):9-27. Disponível em: http:// dx.doi.org/10.5123/S1679-49742013000100002

2. Ministério da Saúde (BR). Programa nacional de imunizações: 40 anos [Internet]. Brasília: Ministério da Saúde; 2013 [citado 2018 mar 29]. Disponível em: https://www.saude.gov.br/svs

3. Temporão JG. 0 Programa Nacional de Imunizações (PNI): origens e desenvolvimento. Hist Ciênc Saúde Manguinhos [Internet]. 2003 [citado 2020 dez 1];10(suppl 2):601-17. Disponível em: https:// doi.org/10.1590/\$0104-59702003000500008

4. Ministério da Saúde (BR). Manual do Sistema de Informação do Programa Nacional de Imunizações: SI-PNI. Brasília: Ministério da Saúde; 2016.

5. Silva BS, Coelho HV, Cavalcante RB, Oliveira VC, Guimarães EAA. Estudo de avaliabilidade do Sistema de Informação do Programa Nacional de Imunização. Rev Bras Enferm [Internet]. 2018 [citado $2020 \mathrm{dez}$ 1];71(supl 1):660-9. Disponível em: https://doi.org/10.1590/0034-7167-2017-0601

6. Ministério da Saúde (BR). Agência Nacional de Vigilância Sanitária - Anvisa. Resolução da Diretoria Colegiada RDC n 197, de 26 de dezembro de 2017. Dispõe sobre os requisitos mínimos para o funcionamento dos serviços de vacinação humana [Internet]. Brasília: Anvisa, 2017 [citado $2020 \mathrm{dez}$ 1]. Disponível em:
0 atendimento a tais recomendações pode qualificar o trabalho, os processos de produção e fluxo das informações do PNI da sala de vacina da localidade mais distante à sede de sua coordenação nacional. $\mathrm{E}$ vice-versa.

\section{Contribuição das autoras}

Todos os autores participaram do desenho do estudo, elaboração e revisão do manuscrito em todas as suas versões, e declaram-se responsáveis por todos os aspectos do trabalho, incluindo a garantia de sua precisão e integridade.

https://sbim.org.br/legislacao/867-rdc-anvisan-197-26-de-dezembro-de-2017

7. Brasil. Ministério da Saúde. Portaria MS/GM n ${ }^{0} 2.363$, de 18 de outubro de 2012. Institui repasse financeiro do Fundo Nacional de Saúde aos Fundos de Saúde dos Estados, Distrito Federal e Municípios, por meio do Piso Variável de Vigilância e Promoção da Saúde, para fomento na implantação do Sistema de Informação do Programa Nacional de Imunizações (SI-PNI) e Sistema de Informação de Agravos de Notificação (SINAN), no âmbito das unidades de saúde [Internet]. Diário Oficial da União, Brasília (DF), 2012 out 19 [citado 2018 ago 29];Seção 1:24. Disponível em: http://bvsms.saude.gov. br/bvs/saudelegis/gm/2012/prt2363_18_10_2012.html

8. Brasil. Ministério da Saúde. Portaria MS/GM n ${ }^{0} 2.984$, de 27 de dezembro de 2016. Revisa a relação de metas e seus respectivos indicadores do Programa de Qualificação das Ações de Vigilância em Saúde(PQAVS) a partir de 2017 [Internet]. Diário Oficial da União, Brasília (DF), 2016 dez 28 [citado 2018 jun 6];Seção 1:109. Disponível em: http://bvsms.saude.gov.br/bvs/ saudelegis/gm/2016/prt2984_27_12_2016.html

9. Centers for Disease Control and Prevention - CDC. Updated guidelines for evaluating public health surveillance systems [Internet]. Washington, D.C.: CDC; 2001 [cited 2018 Mar 29]. Available from: https:/www. cdc.gov/mmwr/preview/mmwrhtml/rr5013a1.htm

10. Ministério da Saúde (BR). Secretaria de Vigilância em Saúde. Manual de normas e procedimentos para vacinação [Internet]. Brasília: Ministério da Saúde; 2014 [citado 2017 jul 3]. 176 p. Disponível em: http://bvsms.saude.gov.br/bvs/publicacoes/ manual_procedimentos_vacinacao.pdf 
11. Ministério da Saúde (BR). Secretaria de Vigilância em Saúde. Nota informativa n ${ }^{\circ}$ 94, de 2017/ CGPNI/DEVIT/SVS/MST. Orientações e indicação de dose única da febre amarela [Internet]. Brasília: Ministério da Saúde; 2017 [citado 2020 dez 1]. Disponível em: https://www.saude.mg.gov. br/images/noticias_e_eventos/000_2018/01jan-fev-marc-abril/Boletins_AEDES/Nota Informativa 94 Com de Acordo 005.pdf

12. Ministério da Saúde (BR). Nota informativa no 62-SEI/2017-CGPNI/DEVIT/SVS/MS. Orienta os serviços de vacinação para a otimização do uso da vacina HPV quadrivalente, com ampliação temporária da faixa etária [Internet]. Brasília: Ministério da Saúde; 2017 [citado 2020 dez 1]. Disponível em: https:/www.saude.gov.br/images/pdf/2017/ agosto/18/SEI_MS-0290791-Nota-Informativa.pdf

13. Teixeira AMS, Silva AA, Silva AF, Domingues CMAS, Locádio ES, Fantinato FFST, Souza LRO, et al. Avaliação dos indicadores de desempenho da vacinação do Programa Nacional de Imunizações e os desafios para elevar as coberturas vacinais no Brasil. In: Ministério da Saúde (BR). Secretaria de Vigilância em Saúde. Saúde Brasil 2019: uma análise da situação de saúde com enfoque nas doenças imunopreveníveis e na imunização [Internet]. Brasília: Ministério da Saúde; 2019 [citado 2020 dez 1]. p. 370-486. Disponível em: https:/portalarquivos2.saude.gov.br/images/pdf/2019/ dezembro/05/Saude-Brasil-2019-imunizacao.pdf

14. Waldman EA, Sato APS. Trajetória das doenças infecciosas no Brasil nos últimos 50 anos: um contínuo desafio. Rev Saúde Pública [Internet]. 2016 dez [citado $2020 \mathrm{dez}$ 1];50:68. Disponível em: https:// doi.org/10.1590/S1518-8787.2016050000232

15. Domingues CMAS, Woycicki JR, Rezende KS, Henriques CMP. Programa Nacional de Imunização: a política de introdução de novas vacinas. Rev Eletr Gestão Saúde [Internet]. 2015 out [citado $2020 \mathrm{dez}$ 1];6(supl 4):3250-74. Disponível em: https://periodicos. unb.br/index.php/rgs/article/view/3331/3017

16. Sato APS. Programa Nacional de Imunização: sistema informatizado como opção a novos desafios. Rev Saúde Pública [Internet]. 2015 jul [citado 2019 fev 6];49:39. Disponível em: https:// doi.org/10.1590/S0034-8910.2015049005925

17. Machado Costa MR, Barbosa J. Situação atual da febre amarela no Brasil. Bol Eletr Epidemiol [Internet]. Brasília: Funasa; 2001 [citado 2020 dez 1]. Disponível em: www.funasa.gov.br
18. Valente NTZ, Fujino A. Atributos e dimensões de qualidade da informação nas ciências contábeis e na ciência da informação: um estudo comparativo. Perspect Ciência Inf [Internet]. 2017 abr-jun [citado 2020 dez 1];21(2):141-67. Disponível em: https://doi.org/10.1590/1981-5344/2530

19. Lima CRA, Schramm JMA, Coeli CM, Silva MEM. Revisão das dimensões de qualidade dos dados e métodos aplicados na avaliação dos sistemas de informação em saúde. Cad Saúde Pública [Internet]. 2009 out [citado 2020 dez 1];25(10):2095-109. Disponível em: https://www.scielo.br/pdf/csp/v25n10/02.pdf

20. Correia LO, Padilha BM, Vasconcelos SML. Métodos para avaliar a completitude dos dados dos sistemas de informação em saúde do Brasil: uma revisão sistemática. Ciênc Saúde Coletiva [Internet]. 2014 [citado $2020 \mathrm{dez}$ 1];19(11):446778. Disponível em: https://www.scielo.br/pdf/ csc/v19n11/1413-8123-csc-19-11-4467.pdf

21. Luhm KR, Waldman EA. Sistemas informatizados de registro de imunização: uma revisão com enfoque na saúde infantil. Epidemiol Serv Saúde [Internet]. 2009 jan-mar [citado $2020 \mathrm{dez}$ 1];18(1):65-78. Disponível em: http://scielo.iec.gov.br/pdf/ess/v18n1/v18n1a07.pdf

22. Teixeira AMS, Mota ELA. Denominators for vaccine coverage estimates: a database study to estimate the population less than one year of age. Epidemiol Serv Saúde [Internet]. 2010 Jul-Sep [cited 2019 Feb 12];19(3):187-203. Available from: http:// dx.doi.org/10.5123/S1679-49742010000300002

23. Ministério da Saúde (BR). Organização PanAmericana da Saúde - OPAS. Fundação Oswaldo Cruz - Fiocruz. A experiência brasileira em sistemas de informação em saúde: falando sobre os sistemas de informação em saúde no Brasil [Internet]. Brasília: Ministério da Saúde; 2009 [citado 2020 dez 1]. 2 v. (Série B. Textos Básicos de Saúde). Disponível em: https://bvsms.saude.gov.br/bvs/publicacoes/ experiencia_brasileira_sistemas_saude_volume2.pdf

24. Tauil MC, Sato APS, Costa ÂA, Inenami M, Ferreira VLR, Waldman EA. Vaccination coverage according to doses received and timely administered based on an electronic immunization registry, Araraquara-SP, Brazil, 2012-2014. Epidemiol Serv Saúde [Internet]. 2017 0ct-Dec [cited 2020 Dez 1];26(4):835-46. Available from: https:// doi.org/10.5123/S1679-49742017000400014 


\section{Abstract}

Objective: To evaluate the National Immunization Program Immunization Surveillance System, based on its Vaccination Record module, for Brazil in 2017. Methods: This was a descriptive study using the Guidelines for Evaluating Public Health Surveillance Systems, published by the Centers for Disease Control and Prevention (CDC/Atlanta/GA/United States) to evaluate the attributes of simplicity, flexibility, data quality, sensitivity, timeliness and usefulness of the system for six vaccines on the child immunization schedule. Results: The Immunization Surveillance System was considered complex in its description; flexible to changes in the immunization schedule; of poor data quality for the DTP and rotavirus vaccines; regular acceptability; high sensitivity for the BCG vaccine; untimely for the bepatitis $B$ vaccine and useful for the purposes of the National Immunization Program. Conclusion: The data quality, acceptability and timeliness results were not satisfactory, so that actions are needed to enhance the information system.

Keywords: Immunization; National Immunization Program; Public Health Surveillance; Program Evaluation; Information Systems; Data Accuracy.

\section{Resumen}

Objetivo: Evaluar el sistema de vigilancia en inmunizaciones, considerando el módulo de Registro del Vacunado del Sistema de Información del Programa Nacional de Inmunizaciones de Brasil, en 2017. Métodos: Estudio descriptivo, utilizando las Guidelines for Evaluating Public Health (CDC/Atlanta) para evaluar los atributos simplicidad, flexibilidad, calidad de los datos, sensibilidad, oportunidad y utilidad del sistema para seis vacunas del calendario de vacunación de niños. Resultados: El sistema de vigilancia fue considerado complejo en su descripción; flexible a los cambios del calendario de vacunación; baja calidad de los datos para la DTP y rotavirus; aceptación regular con alta sensibilidad para la vacuna BCG e inoportuno para la vacuna contra bepatitis $B, y$ útil para las finalidades del Programa Nacional de Inmunizaciones. Conclusión: Algunos atributos, calidad de los datos, aceptabilidad y oportunidad no presentaron resultados satisfactorios, evaluarlos es de extrema importancia para tomar acciones que mejoren la calidad del sistema de vigilancia em inmunizaciones.

Palabras clave: Inmunización; Programas de Immunización; Vigilancia en Salud Pública; Evaluación de Programas y Proyectos de Salud; Sistemas de Información; Calidad de los Datos.

Recebido em 03/02/2020

Aprovado em 09/10/2020

Editora associada: Luciana Guerra Gallo - ㅇ orcid.org/0000-0001-8344-9951 


\title{
Errata
}

No artigo "Avaliação do Sistema de Vigilância do Programa Nacional de Imunizações - Módulo Registro do Vacinado, Brasil, 2017", com número de doi: 10.1590/s1679-49742021000100028, publicado no periódico Epidemiologia e Serviços de Saúde, 30(1):e2019596, na página 14:

Onde se lia:

\begin{abstract}
"Abstract
Objective: To compare structure and work process in Primary Care for implementing medical teleconsultation in municipalities in different regions and with different population sizes (100,000 inhabitants). Methods: This was a cross-sectional study, with descriptive and bivariate analysis, using data from 2017-2018 to assess availability of computers with internet access, webcam, microphone, speaker, as well as to assess team work processes (use of Telessaúde [Telehealth], service supply and demand control center and communication flow). Results: 30,346 primary health centers and 38,865 teams were evaluated. Presence of teleconsultation equipment in the health centers ranged from $1.2 \%$ in large northern municipalities to $26.7 \%$ in small southern municipalities. Established work process ranged from $10.7 \%$ in small northern municipalities to $39.5 \%$ in large southern municipalities. Compared to the South, medium-sized municipalities in the North $(\mathrm{OR}=0.14-95 \% \mathrm{CI} 0.11 ; 0.17)$ and Northeast $(\mathrm{OR}=0.21-95 \% \mathrm{CI} 0.18 ; 0.25)$ regions were less likely to have the necessary equipment. Conclusion: Significant regional inequalities call for investments in Digital Health.

Keywords: Telemedicine; Remote Consultation; Information Technology; Policy Making; Cross-Sectional Studies.
\end{abstract}

\section{Resumen}

Objetivo: Comparar estructura y proceso de trabajo en atención primaria para implementar la teleconsulta médica en municipios de diferentes regiones y tamaños (mil habitantes: 100). Métodos: Estudio transversal, con análisis descriptivo y bivariado, datos de 2017-2018 para evaluar la disponibilidad de computadora con internet, cámara, micrófono, altavoz y proceso de trabajo (uso de Telesalud, centro de regulación central y flujo de comunicación). Resultados: Se analizaron 30.346 unidades y 38.865 grupos. La presencia de equipos de teleconsulta osciló entre $1,2 \%$ en los grandes municipios del Norte y $26,7 \%$ en pequeños municipios del Sur. El proceso de trabajo osciló entre $10,7 \%$ en pequeños municipios del Norte y $39,5 \%$ en grandes municipios del Sur. En comparación con el Sur, municipios medianos del Norte $(\mathrm{OR}=0,14-\mathrm{IC} 95 \% 0,11 ; 0,17)$ y Nordeste $(\mathrm{OR}=0,21-\mathrm{IC} 95 \% 0,18 ; 0,25)$ tenían menos probabilidades de contar con los equipos necesarios. Conclusión: Existe la necesidad de inversiones en Salud Digital, con desigualdades regionales relevantes.

Palabras clave: Telemedicina; Consulta Remota; Tecnología de la Información; formulación de Políticas; Estudios Transversales"

Leia-se:

\begin{abstract}
"Abstract
Objective: To evaluate the National Immunization Program Immunization Surveillance System, based on its Vaccination Record module, for Brazil in 2017. Methods: This was a descriptive study using the Guidelines for Evaluating Public Health Surveillance Systems, published by the Centers for Disease Control and Prevention (CDC/Atlanta/GA/United States) to evaluate the attributes of simplicity, flexibility, data quality, sensitivity, timeliness and usefulness of the system for six vaccines on the child immunization schedule. Results: The Immunization Surveillance System was considered complex in its description; flexible to changes in the immunization schedule; of poor data quality for the DTP and rotavirus vaccines; regular acceptability; high sensitivity for the BCG vaccine; untimely for the hepatitis B vaccine and useful for the purposes of the National Immunization Program. Conclusion:
\end{abstract}


The data quality, acceptability and timeliness results were not satisfactory, so that actions are needed to enhance the information system.

Keywords: Immunization; National Immunization Program; Public Health Surveillance; Program Evaluation; Information Systems; Data Accuracy."

\section{Resumen}

Objetivo: Evaluar el sistema de vigilancia en inmunizaciones, considerando el módulo de Registro del Vacunado del Sistema de Información del Programa Nacional de Inmunizaciones de Brasil, en 2017. Métodos: Estudio descriptivo, utilizando las Guidelines for Evaluating Public Health (CDC/Atlanta) para evaluar los atributos simplicidad, flexibilidad, calidad de los datos, sensibilidad, oportunidad y utilidad del sistema para seis vacunas del calendario de vacunación de niños. Resultados: El sistema de vigilancia fue considerado complejo en su descripción; flexible a los cambios del calendario de vacunación; baja calidad de los datos para la DTP y rotavirus; aceptación regular con alta sensibilidad para la vacuna BCG e inoportuno para la vacuna contra hepatitis B, y útil para las finalidades del Programa Nacional de Inmunizaciones. Conclusión: Algunos atributos, calidad de los datos, aceptabilidad y oportunidad no presentaron resultados satisfactorios, evaluarlos es de extrema importancia para tomar acciones que mejoren la calidad del sistema de vigilancia em inmunizaciones.

Palabras clave: Inmunización; Programas de Immunización; Vigilancia en Salud Pública; Evaluación de Programas y Proyectos de Salud; Sistemas de Información; Calidad de los Datos. 